\title{
Recurrent arterial thrombosis of the lower extremity with secondary thrombocythemia due to reperfusion injury: a case report
}

\author{
Yulan Geng', Zhengli Chen ${ }^{1}$, Lili Dai ${ }^{1}$, Guochao Liu ${ }^{2}$, Xinqi $\mathrm{He}^{3}$ \\ ${ }^{1}$ Department of Laboratory Medicine, the First Hospital of Hebei Medical University, Shijiazhuang, China; ${ }^{2}$ Shenzhen Mindray Bio-Medical \\ Electronic Co., Ltd., Shenzhen, China; ${ }^{3}$ Department of Vascular Surgery, the First Hospital of Hebei Medical University, Shijiazhuang, China \\ Correspondence to: Xinqi He, MD. Department of Vascular Surgery, the First Hospital of Hebei Medical University, 89 Donggang Road, Shijiazhuang \\ 050031, China. Email: hexinqi192@126.com.
}

\begin{abstract}
Thrombocythemia is an important cause for thrombogenesis and can be classified as essential or secondary according to the etiology. Secondary thrombocythemia (ST), also called reactive thrombocytosis, is caused by a disorder that triggers increased production by normal platelet-forming cells and is characterized in terms of abnormal increased number of platelet in blood and megakaryocytes in bone marrow. Previous reports have found that complications from malignant tumors, chronic inflammation, acute inflammation, acute hemorrhage, spleen resection etc. to be the common causes of ST. A 53-year-old Chinese male with right lower limb arterial ischemic embolism developed recurring arterial thrombosis at the previous site after operation. During his hospitalization, the patient had a platelet count that was positively correlated with alanine transaminase (ALT), aspartate aminotransferase (AST), lactate dehydrogenase (LDH), $\alpha$-hydroxybutyrate dehydrogenase $(\alpha-\mathrm{HBDH})$, creatine kinase $(\mathrm{CK})$, and creatine kinase isoenzyme $\mathrm{MB}$ (CK-MB) while his thromboelastogram (TEG) and platelet aggregation test obtained by sequential platelet count showed inconsistent platelet function. We describe a case in which ischemia-reperfusion injury caused ST and recurrent thrombosis and analyse the probable cause of contradictory results of different platelet function tests. In thrombolytic therapy, we recommend adding platelet count and two more platelet aggregation tests to the routine laboratory items to aid in the prevention of recurrent thrombosis.
\end{abstract}

Keywords: Secondary thrombocythemia (ST); recurrent thrombosis; reperfusion injury; platelet aggregation function; case report

Submitted Jul 13, 2020. Accepted for publication Sep 11, 2020.

doi: 10.21037/apm-20-1649

View this article at: http://dx.doi.org/10.21037/apm-20-1649

\section{Introduction}

Thrombocythymia is an important cause for thrombogenesis and is characterized by peripheral platelet count more than the upper limit of normal or more than $450 \times 10^{9} / \mathrm{L}$ (1), affecting one fifth of trauma patients and up to one third of patients in intensive care unit $(2,3)$. Thrombocytosis predicts an underlying solid tumor diagnosis in $11.6 \%$ of men and $6.5 \%$ of women (4) while pretreatment thrombocytosis was significantly related to a decreased overall survival and disease-free survival (5). The condition can be classified as primary or essential thrombocytosis resulting from a clonal bone marrow abnormality such as myeloproliferative neoplasm (MPN), secondary thrombocytosis (ST) and inherited thrombocytosis according to the etiology (1). The most common form of thrombocytosis is ST. ST, also known as reactive thrombocytosis, is caused by a disorder that triggers increased production by normal platelet-forming cells. It is known to commonly result from malignant tumors, chronic inflammation, acute inflammation, acute hemorrhage, spleen resection, and other conditions. Most patients with ST show no symptoms, but a few, 
usually older patients, have atherosclerosis $(6,7)$. In the case discussed here, our patient developed recurring arterial thrombosis at the previous site of his right lower extremity artery after balloon-catheter thrombectomy in the period of hospitalization. During his admission, his thromboelastogram (TEG) and platelet aggregation test showed inconsistent platelet function, while laboratory examinations showed that the patient's platelet (PLT) count and concentrations of alanine transaminase (ALT), aspartate aminotransferase (AST), lactate dehydrogenase (LDH), $\alpha$-hydroxybutyrate dehydrogenase $(\alpha-\mathrm{HBDH})$, creatine kinase $(\mathrm{CK})$, and creatine kinase isoenzyme $\mathrm{MB}$ (CK$\mathrm{MB})$ were fluctuating. However, following the laboratory findings came to normality, the patient's thrombosis disappeared, and he was discharged.

We present the following article in accordance with the CARE reporting checklist (available at http://dx.doi. org/10.21037/apm-20-1649).

\section{Case presentation}

A 53-year-old Chinese male presented to our hospital with a 2-year history of intermittent claudication that limited his ability to walk 300 meters and a 4-hour history of sudden pain and numbness. Physical examination found no abnormalities of the heart, lung, or abdomen. Both lower limbs had normal length and shape. His bilateral femoral artery pulse was palpable, with the right one being weaker. The 10-cm distal skin of his right knee was cool and pale, while his right ankle and foot had hypoesthesia. Laboratory findings were normal for complete blood cell count, coagulation, liver function, renal function, cholesterol, and blood glucose. Doppler ultrasound (DUS) showed no vascular blood flow in the right acrotarsium and posterior tibial artery, and echogenic content was consistent with embolism. Computed tomography (CT) revealed bilateral femoral artery thrombosis. According to the presenting symptoms, signs, and imaging findings, he was diagnosed with right lower limb arterial ischemic embolism and thrombosis, with-rest pain of the right limb. Under general anesthesia, the patient underwent right lower limb artery balloon-catheter thrombectomy and right iliac artery stent implantation which resulted in good distal perfusion. This was followed with conventional administration with continuous heparin for anticoagulation and urokinase for thrombolysis in order to avoid recurrent thrombosis.
Routine laboratory tests and physical examination monitored the antithrombotic effect.

The patient was initially stable, but on postoperative day 3, began to have cool skin of the right lower limb extremity, which was confirmed with DUS as right femoral artery thrombosis at the previous site. An emergency reoperation was performed for resolution of the thrombosis. To determine the cause of patient's excessive thrombotic tendency before balloon-catheter thrombectomy, an additional laboratory review was conducted and showed that he had higher PLT, ALT, AST, LDH, $\alpha-H B D H, C K$, and $\mathrm{CK}-\mathrm{MB}$ than baseline levels (Figure 1). Clopidogrel and aspirin were added to the therapeutic schedule for anti-platelet aggregation. On postoperative day 5, an antiplatelet drug effect was evaluated with a platelet aggregation test obtained by sequential platelet count (Aggrestar PL16, Sinnowa, China) and TEG (Thrombelastography, Haemoscope, US). His platelet aggregation test (Figure 2) and TEG (Figure 3) showed unusually inconsistent platelet function. The platelet aggregation test by counting platelet number revealed that the platelet aggregation rate was $54.4 \%$ for arachidonic acid (AA)-induced antiplatelet effect of aspirin and $15.2 \%$ for adenosine diphosphate (ADP)induced antiplatelet effect of $\mathrm{P} 2 \mathrm{Y} 12$ inhibitor of clopidogrel, which suggested that the inhibition rate of AA and ADP were $45.6 \%$ and $84.8 \%$, respectively. Meanwhile TEG showed no inhibition effects for AA- and ADP-induced antiplatelet agents, the inhibition rates being 0 . Later that same day, after heparin was switched to argatroban and clopidogrel and aspirin were switched to ticagrelor, his thrombosis symptoms gradually disappeared. It was very lucky that there were no complications such as bleeding and hyperkalemia during antithrombotic therapy. From postoperative day 2 onward, the patient showed persistent thrombosis at the same site of his right lower extremity artery while his PLT, ALT, AST, LDH, and CK fluctuated up and down (Figure 1). During the hospitalization, his PLT count was positively correlated to ALT, AST, LDH, HBDH, CK, and CK-MB $(r=0.4442,0.6303,0.4892$, $0.4622,0.6154,0.4618$, respectively). On postoperative day 23 , his symptoms and laboratory results showed no obvious abnormalities, and the patient was discharged.

Written informed consent was obtained from the patient for publication of this study and any accompanying images. And all procedures performed in this study was in accordance with the ethical standards of the institutional 


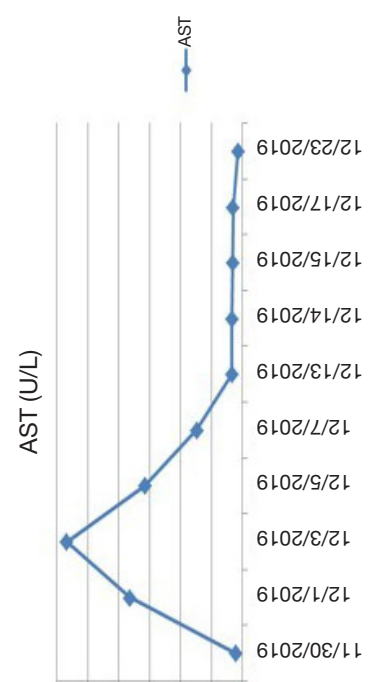

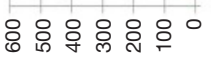
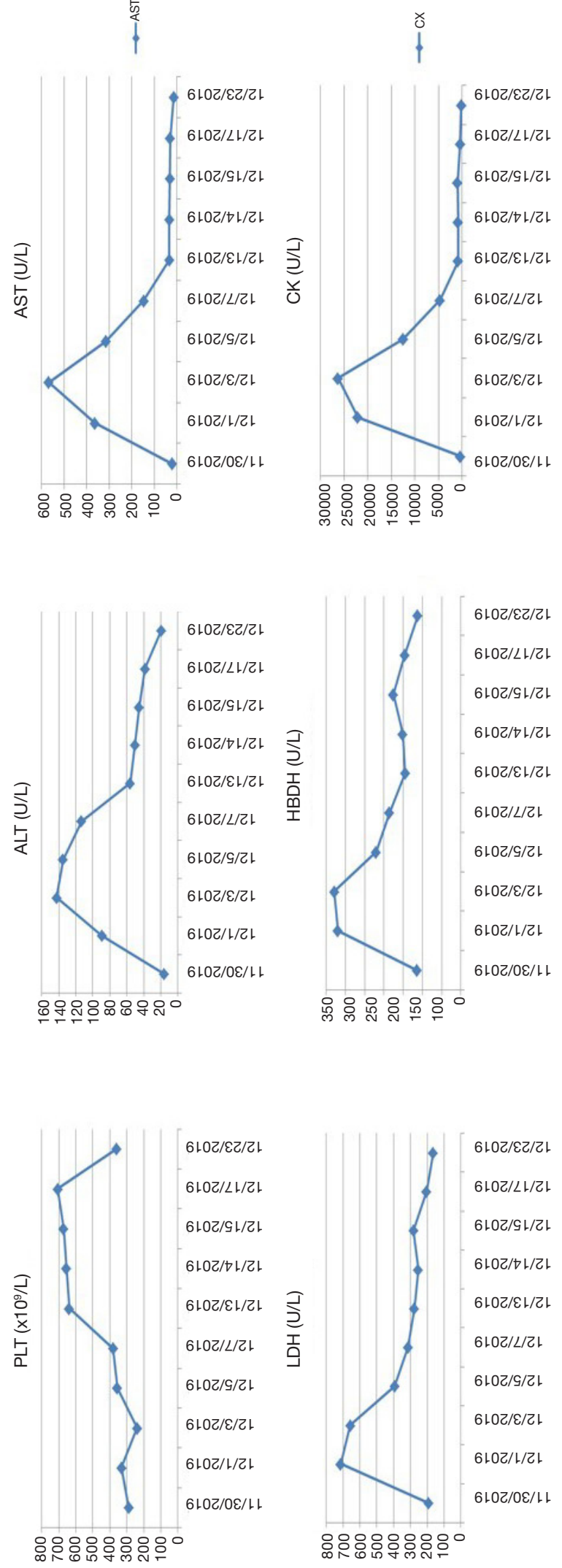

(C) Annals of Palliative Medicine. All rights reserved.

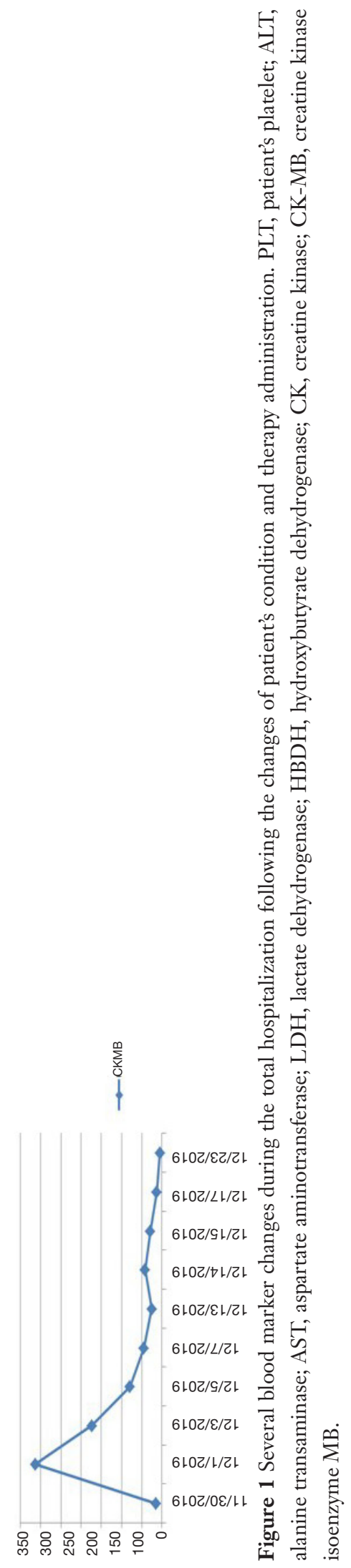




\begin{tabular}{|c|c|c|c|c|c|c|c|c|c|c|c|c|c|}
\hline \multicolumn{7}{|c|}{ Platelet Aggregation Test (ADP) } & \multicolumn{7}{|c|}{ Platelet Aggregation Test (AA) } \\
\hline \multirow{2}{*}{$\begin{array}{c}\text { Serial lio. } \\
1\end{array}$} & \multirow{2}{*}{$\begin{array}{c}\text { lems } \\
\text { PLT-0 }\end{array}$} & \multirow{2}{*}{$\begin{array}{l}\text { Results } \\
626\end{array}$} & \multirow{2}{*}{$10^{\wedge} 9 / \mathrm{L}$} & \multicolumn{2}{|c|}{ Reference values } & \multirow{2}{*}{$\begin{array}{l}\text { Hint } \\
\uparrow \uparrow\end{array}$} & \multirow{3}{*}{$\begin{array}{l}\text { Seriallio. } \\
1\end{array}$} & \multirow{3}{*}{$\begin{array}{c}\text { lems } \\
\text { PLI-0 }\end{array}$} & \multirow{3}{*}{$\begin{array}{l}\text { Resuls } \\
626\end{array}$} & \multirow{3}{*}{$\begin{array}{c}\text { Unt : } \\
10^{\wedge} \mathrm{g} / \mathrm{L}\end{array}$} & \multirow{2}{*}{\multicolumn{2}{|c|}{ Reterenevaluss }} & \multirow{3}{*}{$\begin{array}{l}\text { Hint } \\
\uparrow \uparrow\end{array}$} \\
\hline & & & & 100 & 300 & & & & & & & & \\
\hline 2 & $M P V-0$ & 7.16 & $\mathrm{fL}$ & 7.00 & 11.00 & & & & & & 100 & 300 & \\
\hline 3 & $\mathrm{PDH}-0$ & 8.78 & $\mathrm{fL}$ & 7.00 & 17.00 & & 2 & MPV-0 & 7.16 & fL & 7.00 & 11.00 & \\
\hline 4 & MAR & 17.1 & 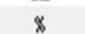 & 30.0 & 70.0 & $\downarrow$ & 3 & PDW -0 & 8.78 & fL & 7.00 & 17.00 & \\
\hline 5 & MAT & 545 & s & & & & 4 & MAR & 56.2 & 8 & 40.0 & 80.0 & \\
\hline 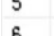 & MAI & 545 & $s$ & 210 & 600 & & 5 & MAT & 487 & s & 210 & 600 & \\
\hline 6 & hAR & 15.2 & $\$$ & 20 & 60 & $\downarrow$ & 6 & HAR & 54.4 & $x$ & 30 & 70 & \\
\hline 7 & $\mathrm{RBC}-0$ & 2.66 & $10^{\wedge} 12 / \mathrm{L}$ & 3.50 & 5.50 & $\downarrow$ & 7 & $\mathrm{RBC}-0$ & 2.66 & $10^{\wedge} 12 / \mathrm{L}$ & 3.50 & 5.50 & $\downarrow$ \\
\hline 8 & $M C V-0$ & 89 & $\mathrm{fL}$ & 80 & 100 & & 8 & $M C V-0$ & 89 & $\mathrm{fL}$ & 80 & 100 & \\
\hline 9 & $\mathrm{RDH}-0$ & 74.50 & $\mathrm{fL}$ & 35.00 & 56.00 & $\uparrow$ & 9 & $\mathrm{RDW}-0$ & 74.50 & fL & 35.00 & 56.00 & $\uparrow$ \\
\hline
\end{tabular}

Figure 2 Platelet aggregation test revealed that part platelet function was inhibited. PLT-0, initial Platelet count; MPV-0, initial mean platelet volume; PDW-0, initial platelet distribution width; MAR, maximum platelet aggregation ratio; MAT, maximum platelet aggregation time; AAR, average platelet aggregation rate; RBC-0, initial red blood cell count; MCV-0, initial mean red blood cell volume; RDW-0, initial red blood cell distribution width.
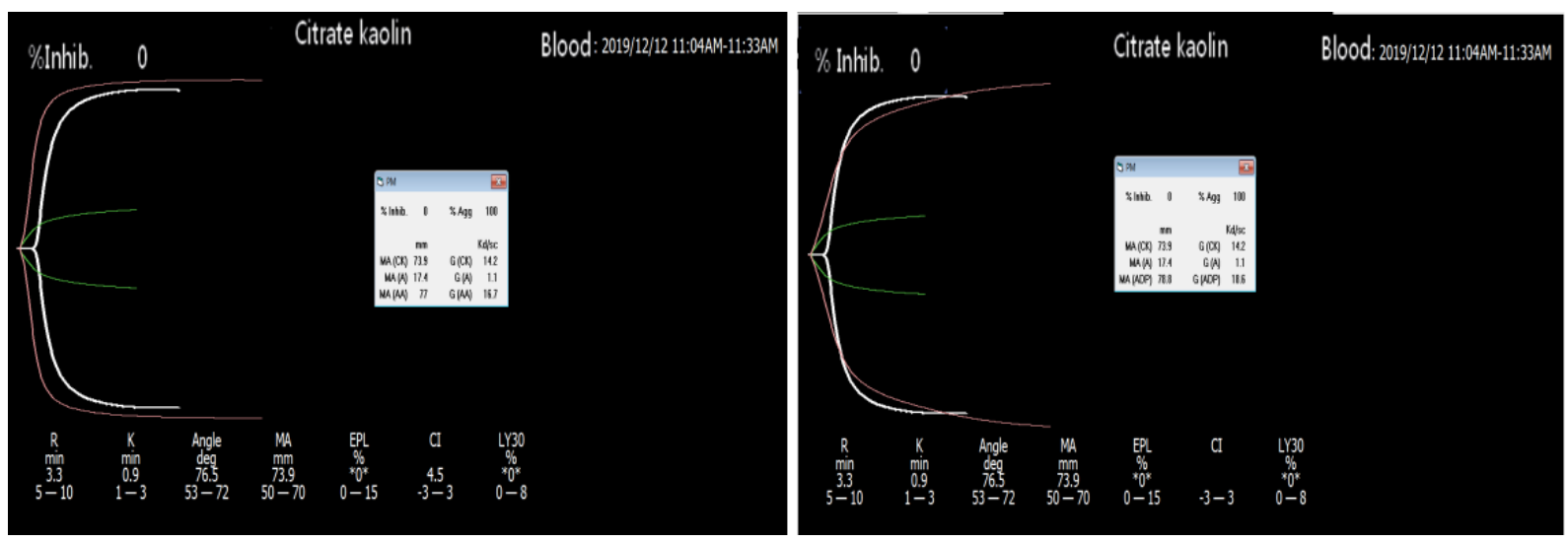

Figure 3 TEG showed no platelet inhibition of AA-induced antiplatelet effect for aspirin and ADP-induced antiplatelet effect for clopidogrel in the patient. TEG, thromboelastogram; AA, arachidonic acid; ADP, adenosine diphosphate.

and/or national research committee(s) and with the Helsinki Declaration (as revised in 2013).

\section{Discussion}

Peripheral arterial disease (PAD) is a progressive disease most often resulted from atherosclerosis and characterized by obstruction of arterial blood flow in the lower limbs. Lower limb peripheral artery disease (PAD) is highly prevalent throughout the world. Anyway, it is uncommon before the age of 50 years. A higher prevalence of PAD among Americans 60 years and older has been reported to range from $12 \%$ to $20 \%$. Strikingly, the rate for African Americans was about twice that of non-Hispanic whites at any given age (8). Furthermore, the National Health and
Nutrition Examination Survey (NHANES) has estimated that the prevalence of PAD to be $4.3 \%$ among adults aged 40 years and over and $14.5 \%$ among those aged 70 years of age and over (9). Global data on trends in PAD prevalence between the years 2000 and 2010 demonstrated that the number of individuals with PAD increased by $28.7 \%$ in low-income and middle-income countries and by $13.1 \%$ in high-income countries (10). During the past decades, the consensus-based venous thrombosis mechanism was the combination of stasis and hypercoagulability, much more than endothelial damage, and less by platelets. Instead, platelets are essential for primary haemostasis, repair of damaged endothelium and play an important role in the arterial thrombosis. Inflammation, lipids and the immune system, through a complex interplay, are also 
vital contributors to arterial thrombosis (11). Cigarette smoking, diabetes mellitus, hypertension, dyslipidemia, obesity, alcohol consumption, race, homocysteine, C-reactive protein, fibrinogen, chronic kidney disease, genetic factors, and so on are probable risk factors of PAD, although the onset of PAD is often asymptomatic and, in any case (12). Critical lower limb ischemia (CLI) is a manifestation of PAD that is seen in patients with typical chronic ischemic rest pain or with ischemic skin lesions, ulcers, or gangrene persisting longer than 2 weeks. Medical interventions are important to improve arterial perfusion. In the lower extremities, PAD affects three major arterial segments including the femoropopliteal arteries. Among the therapeutic options available, surgical revascularization is considered superior for resolving acute or limb-threatening ischemia, especially for those patients with inadequate response to the aforementioned therapies (8).

In terms of drug-induced thrombocytosis, there are three types of drugs with relatively high composition: antitumor drugs, blood system drugs and antibacterial drugs. Clinical manifestations: progressive increase of platelets, severe thrombosis, hematoma thrombosis and so on. The mechanism is as follows: promote the secretion of cytokines and induce platelet formation; destroy the regulatory system of PLT production, promote the maturation of megakaryocytes and increase the production of PLT; change gene expression to promote the maturation of megakaryocytes $(13,14)$.

During perioperative periods, thrombosis is a major cause of morbidity and, even with anticoagulant therapy, is reported to be as high as $36 \%$ (15). Although advanced age, pregnancy, surgery, immobilization, malignancy, anticoagulation resistance, and oral contraceptive use are considered to be important clinical risk factors, the cause of many venous thrombotic events remains unknown (16). However, due to the rarity of arterial thromboembolism (aTE) and the inherent limitations imposed by the Taiwan registry data (17), few studies have explored the recurrence of arterial thrombosis. It has been reported that BMI $>23 \mathrm{~kg} / \mathrm{m}^{2}$, hypertension, cerebrovascular disease, atrial fibrillation, stage IV disease, and AST level are risk factors of aTE. Among these, however, only hypertension, atrial fibrillation, and high AST level have proven to be independent risk factors (18). The patient presented here also had arterial thrombosis of the right lower limb. Unfortunately, his arterial thrombosis recurred. A case report demonstrated that the drug of hydroxyurea promoted secondary severe thrombocytosis in a patient with hereditary spherocytosis after splenectomy, but no literatures showed that antithrombotic drugs can cause ST (19). We were convinced that no injury occurred during surgical procedure, and we concluded that arterial thrombosis recurred due to reperfusion mechanisms. This phenomenon is mentioned in some literature and is due to oxidative stress, although the exact cause is not known $(20,21)$.

After the first operation, the patient's PLT count was markedly increased, indicating thrombocytosis. However, thrombopoiesis occurs in the bone marrow, and it is regulated by the hormone, thrombopoietin (TPO). Thrombocytosis may be a secondary phenomenon, reflecting an inflammatory state, infection, iron deficiency, recent surgery, haemolysis, or resulting from underlying neoplasms while increased concentration of peripheral thrombopoietin and pro-inflammatory cytokines (for example IL-6) are included in the pathogenesis of ST (22). This case was clearly secondary thrombocythemia (ST) because the patient improved after his PLT count returned to normal.

Ischemia-reperfusion injury is a well-known pathological condition which may lead to disability and mortality (23). Tissue injury and/or death occur as a result of the initial ischemic events, and are primarily caused by the magnitude and duration of the interruption in the blood supply, and the subsequent damage induced by reperfusion (24). The gut, lung, liver, kidney, skeletal muscle, heart, and liver are the commonly injured organs (25). We found there to be a moderate to high correlation between PLT count and the levels of ALT, AST, LDH, $\alpha-\mathrm{HBDH}, \mathrm{CK}$, and CK-MB. It is generally agreed that ALT and AST reflect liver function while $\mathrm{LDH}, \alpha-\mathrm{HBDH}, \mathrm{CK}$, and CK-MB reflect myocardial function. During reperfusion, liver cells produce not only more ALT and AST but also more TPO which is the primary and vital regulator of megakaryocyte progenitor expansion and differentiation (26). No reports from the literature suggest that myocardial injury contributes to PLT production, although our results showed a slight relationship between PLT count and myocardial markers. Moreover, none of the current antiplatelet therapeutics protect against reperfusion injury, which is defined as myocardial injury caused by reoxygenation of a previously ischemic myocardium (27). Reperfusion injury accounts for upwards of $50 \%$ of the final size of a myocardial infarct and is characterized by impaired microvascular 
perfusion (27). Some studies found the levels of both serum malondialdehyde and glutathione to be significantly higher in a lower limb ischemia-reperfusion group and liver ischemia-reperfusion group, while the damage score from histological evaluation was found to be higher in a liver ischemia-reperfusion group and lower limb ischemiareperfusion group (28). Our case showing an abnormally high platelet count and having other blood makers gradually return to normal, suggests that reperfusion injury is a key cause of ST and thrombosis. Moreover, one study found that ST was associated with in vitro prothrombotic tendency correlated to thrombin generation, thrombin activity level depending on coagulation factors. Patients with moderate-to-severe thrombocytosis (platelet count $>700 \times 10^{9} / \mathrm{L}$ ) have significantly higher fibrinogen, factor VII, and VWF antigen and activity level than those with mild thrombocytosis (platelet count $500-700 \times 10^{9} / \mathrm{L}$ ) (29). It is suggested that using both antiplatelet drug and anticoagulants may be a better way for prevention and treatment of ST, especially after postoperative reperfusion.

In order to evaluate antiplatelet effect of aspirin and clopidogrel, AA and ADP platelet function tests were carried out with TEG and platelet aggregation test by counting platelet number. The results of TEG and platelet aggregation test showed contradictory, the former being 0 of the degree of AA and ADP inhibition, the latter being $45.6 \%$ and $84.8 \%$, respectively. There are 13 methodologies for assessment of platelet function with various methodological principles and each has its own pros and cons (30). This case revealed poor correlations between TEG and platelet aggregation test after a rapid rising postoperative platelet count over $350 \times 10^{9} / \mathrm{L}$, so we speculate that TEG is less sensitive to platelet aggregation function when a patient has a higher platelet number. Gremmel et al. reported that the influencing factors for platelet reactivity during clopidogrel therapy were assaydependent (31). Therefore, in antiplatelet treatment, two more assays each sensitive to targeted drugs are available for evaluating platelet function in order to complement each other pros and cons.

\section{Conclusions}

Although the recurrent thrombogenesis in this case was successfully resolved, careful attention must be paid to the rational administration of ST and it is suggested to add platelet count and two more platelet aggregation tests to the routine laboratory items during thrombolysis.

\section{Acknowledgments}

Funding: None.

\section{Footnote}

Reporting Checklist: The authors have completed the CARE reporting checklist. Available at http://dx.doi.org/10.21037/ apm-20-1649

Conflicts of Interest: All authors have completed the ICMJE uniform disclosure form (available at http://dx.doi. org/10.21037/apm-20-1649). The authors have no conflicts of interest to declare.

Ethical Statement: The authors are accountable for all aspects of the work in ensuring that questions related to the accuracy or integrity of any part of the work are appropriately investigated and resolved. Written informed consent was obtained from the patient for publication of this study and any accompanying images. And all procedures performed in this study was in accordance with the ethical standards of the institutional and/or national research committee(s) and with the Helsinki Declaration (as revised in 2013).

Open Access Statement: This is an Open Access article distributed in accordance with the Creative Commons Attribution-NonCommercial-NoDerivs 4.0 International License (CC BY-NC-ND 4.0), which permits the noncommercial replication and distribution of the article with the strict proviso that no changes or edits are made and the original work is properly cited (including links to both the formal publication through the relevant DOI and the license). See: https://creativecommons.org/licenses/by-nc-nd/4.0/.

\section{References}

1. Kanya P, Rattarittamrong E, Wongtakan O, et al. Platelet Function Tests and Inflammatory Markers for the Differentiation of Primary Thrombocytosis and Secondary Thrombocytosis. Asian Pac J Cancer Prev 2019;20:2079-85.

2. Valade N, Decailliot F, Makarova E, et al. Thrombocytosis after trauma: incidence, aetiology, and clinical significance. 
Br J Anaesth 2005;94:18-23.

3. Banach M, Lautenschläger C, Kellner P, et al. Etiology and clinical relevance of elevated platelet count in ICU patients: A retrospective analysis. Med Klin Intensivmed Notfmed 2018;113:101-7.

4. Bailey SE, Ukoumunne OC, Shephard E, et al. How useful is thrombocytosis in predicting an underlying cancer in primary care? a systematic review. Fam Prac 2017;34:4-10.

5. Nie D, Yang E, Li Z. Pretreatment thrombocytosis predict poor prognosis in patients with endometrial carcinoma: a systematic review and meta-analysis. BMC Cancer 2019; 19:73.

6. Mumford J, Flanagan B, Keber B, et al. Hematologic conditions: platelet disorders. FP Essent 2019;485:32-43.

7. Appleby N, Angelov D. Clinical and laboratory assessment of a patient with thrombocytosis. Br J Hosp Med (Lond) 2017;78:558-64.

8. Kosmas CE, Silverio D, Sourlas A, et al. Role of lipoprotein (a) in peripheral arterial disease. Ann Transl Med 2019;7:S242.

9. Selvin E, Erlinger TP. Prevalence of and risk factors for peripheral arterial disease in the United States: results from the National Health and Nutrition Examination Survey, 1999-2000. Circulation 2004;110:738-43.

10. Fowkes FG, Rudan D, Rudan I, et al. Comparison of global estimates of prevalence and risk factors for peripheral artery disease in 2000 and 2010: a systematic review and analysis. Lancet 2013;382:1329-40.

11. Previtali E, Bucciarelli P, Passamonti SM, et al. Risk factors for venous and arterial thrombosis. Blood Transfus 2011;9:120-38.

12. Crigui MH, Aboyans V. epidemiology of peripheral artery disease. Circulation Research 2016;116:1509-26.

13. Jansen T, Altmeyer P. Thrombocytosis induced by lowdose isotretinoin. Int J Dermatol 2000;39:956-7.

14. Tejaswi C, Mohanan S, Murugaiyan R, et al. Double trouble: Cyclosporine-induced thrombocytosis in a patient with methotrexate toxicity: Are they related? J Pharmacol Pharmacother 2015;6:160-2.

15. Ahmed S, Shahid RK, Bhatt H, et al. Chemotherapyrelated thrombocytosis: does it increase the risk of thromboembolism? Oncology 2012;82:327-32.

16. Gordon RJ, Lombard FW. Perioperative venous thromboembolism: a review. Anesth Analg 2017;125:403-12.

17. Huh IY, Han IS, Lee HK, et al. Recurrent thrombosis after carotid endarterectomy secondary to activated protein $\mathrm{C}$ resistance and essential thrombocytosis. Medicine (Baltimore) 2018;97:44.

18. Chan PC, Chang WL, Hsu MH, et al. Higher stroke incidence in the patients with pancreatic cancer: A nationbased cohort study in Taiwan. Medicine (Baltimore) 2018;97:e0133.

19. Hung YS, Chen JS, Chen YY, et al. Incidence, risk factors, and outcomes of arterial thromboembolism in patients with pancreatic cancer following palliative chemotherapy. Cancers (Basel) 2018;10:432.

20. Sarbay H, Akbayram S. Secondary severe thrombocytosis in a patient who underwent splenectomy due to hereditary spherocytosis and its treatment using hydroxyurea. Pan Afr Med J 2019;32:175.

21. Ferrari RS, Andrade CF. Oxidative Stress and Lung Ischemia-Reperfusion Injury. Oxid Med Cell Longev 2015;2015:590987.

22. Kurian GA, Rajagopal R, Vedantham S, et al. The Role of Oxidative Stress in Myocardial Ischemia and Reperfusion Injury and Remodeling: Revisited. Oxid Med Cell Longev 2016;2016:1656450.

23. Appleby N, Angelov D. Clinical and laboratory assessment of a patient with thrombocytosis. Br J Hosp Med (Lond) 2017;78:558-64.

24. Wu MY, Yiang GT, Liao WT, et al. Current Mechanistic Concepts in Ischemia and Reperfusion Injury. Cell Physiol Biochem 2018;46:1650-67.

25. Peralta C, Jiménez-Castro MB, Gracia-Sancho J. Hepatic ischemia and reperfusion injury: effects on the liver sinusoidal milieu. J Hepatol 2013;59:1094-106.

26. Kalogeris T, Baines CP, Krenz M, et al. Cell biology of ischemia/reperfusion injury. Int Rev Cell Mol Biol 2012;298:229-317.

27. Hitchcock IS, Kaushansky K. Thrombopoietin from beginning to end. Br J Haematol 2014;165:259-68.

28. Yellon DM, Hausenloy DL. Myocardial reperfusion injury. N Engl J Med 2007;357:1121-35.

29. Gökalp G, Eygi B, Kiray M, et al. How important is the damage to the liver after lower limb ischemia-reperfusion? An experimental study in a rat model. Turk Gogus Kalp Damar Cerrahisi Derg 2020;28:127-33.

30. Rottenstreich A, Shai E, Kleinstern G, et al. Assessment of procoagulant potential in patients with reactive thrombocytosis and its association with platelet count. Eur J Haematol 2018;100:286-93.28. Paniccia R, Priora 
R, Liotta AA, et al. Platelet function tests: a comparative review. Vasc Health Risk Manag 2015;11:133-48.

31. Gremmel T, Steiner S, Seidinger D, et al. The influencing factors for clopidogrel-mediated platelet inhibition are assay-dependent. Thromb Res 2011;128:352-7.

(English Language Editor: J. Gray)

Cite this article as: Geng Y, Chen Z, Dai L, Liu G, He X. Recurrent arterial thrombosis of the lower extremity with secondary thrombocythemia due to reperfusion injury: a case report. Ann Palliat Med 2020;9(5):3690-3697. doi: 10.21037/ apm-20-1649 